\title{
LECTURES ON REPRESENTATION THEORY
}


This page is intentionally left blank 


\section{LECTURES ON}

REPRESENTATION

THEORY

\section{JING-SONG HUANG}

Department of Mathematics

The Hong Kong University of Science and Technology Hong Kong 
Published by

World Scientific Publishing Co. Pte. Ltd.

P O Box 128, Farrer Road, Singapore 912805

USA office: Suite 1B, 1060 Main Street, River Edge, NJ 07661

UK office: 57 Shelton Street, Covent Garden, London WC2H 9HE

\author{
Library of Congress Cataloging-in-Publication Data \\ Huang, Jing-Song. \\ Lectures on representation theory / Jing-Song Huang. \\ p. $\mathrm{cm}$. \\ Includes bibliographical references. \\ ISBN 9810237243 -- ISBN 9810237251 (pbk) (alk. paper) \\ 1. Representations of groups
}

QA176.H8 1999

98-51615

$512 \cdot 2--\mathrm{dc} 21$

CIP

\title{
British Library Cataloguing-in-Publication Data
}

A catalogue record for this book is available from the British Library.

Copyright $\odot 1999$ by World Scientific Publishing Co. Pte. Ltd.

All rights reserved. This book, or parts thereof, may not be reproduced in any form or by any means, electronic or mechanical, including photocopying, recording or any information storage and retrieval system now known or to be invented, without written permission from the Publisher.

For photocopying of material in this volume, please pay a copying fee through the Copyright Clearance Center, Inc., 222 Rosewood Drive, Danvers, MA 01923, USA. In this case permission to photocopy is not required from the publisher.

Printed in Singapore. 


\section{Preface}

In this book we expand upon a series of lectures delivered during the Nankai Mathematical Summer School. The purpose of those lectures was to provide an introduction to Lie groups, Lie algebras and their representations as well as to introduce some directions of current research to graduate students who have little specialized knowledge of representation theory.

As we move forward into the next century, science has become essential in every part of our society. Mathematics is the very heart of science because all scientific truth is expressed in mathematical terms. Group symmetry is considered to be the soul of modern mathematics. Representation theory is a fundamental tool for studying group symmetry - geometric, analytic, or algebraic - by means of linear algebra. It plays an important role in many recent developments of mathematics and theoretical physics. Indeed, in his often-quoted statement, Izrail Gelfand remarked, "all of mathematics is some kind of representation theory."

Albert Einstein once said, "everything should be made as simple as possible, but not simpler." We think this should be particularly true for mathematical theories. Therefore we assume here that the reader only has a good knowledge of undergraduate linear algebra and a modest knowledge of undergraduate abstract algebra. The book is organized in such a way that we think it will be easier for a beginner to understand. The book is divided into four parts: (I) finite groups, (II) simple Lie algebras, (III) compact Lie groups and (IV) non-compact Lie groups. Each part consists of three chapters among which the first one is about the structure theory of the corresponding subject, the second one introduces the general theory of the corresponding representations and the third one considers some special, but in my opinion, the most interesting topics of representation theory. The similarities and connections between all these four different parts are quite obvious.

Most of Part I-III of the book consists of relatively elementary material of representation theory. These theories are natural continuation of basic linear algebra and the reader will find applications in many fields of mathematics and physics. We have not attempted to give the most complete proofs but to convey the essential ideas of representation theory. Useful references are provided so 
that the interested readers can find the detailed proofs elsewhere. Part I deals with finite groups which do not require any Lie theory. We use the symmetric group $S_{n}$ as an example to show the characters of group representations. Part II is about the structure and representation theory of simple Lie algebras over C. Part III considers the representations of compact Lie groups and applications. The last chapter of Part III contains recent results on extension of Weyl's constructions. Part IV covers some advanced material on infinite-dimensional representations of non-compact groups. We use the standard example, the representations of $S L(2, \mathbb{R})$. We have also included the theory of coadjoint nilpotent orbits and minimal representations of complex simple groups. An alternative approach to an introduction of infinite-dimensional representations that was presented in the lectures at Nankai University is the representations of the general Linear group $G L(n, \mathbb{R})$ and its universal covering group $\widetilde{G L}(n, \mathbb{R})$. The theory of complete classifications of irreducible unitary representations of $G L(n, \mathbb{R})$ and of $\overline{G L}(n, \mathbb{R})$ together with the lifting of the unitary characters which relates the two groups is extremely appealing. However, we have included these materials here to keep this introductory book a reasonable length. Interested readers can refer to three journal articles [V2], [H1] and [AH]. The last part of the book, though short, touches on almost all the important ideas of current research such as orbit methods, minimal representations and dual pair correspondences.

In his famous lecture delivered at the International Congress of Mathematicians in 1900, David Hilbert quoted an unnamed mathematician, "a mathematical theory is not to be considered complete until you have made it so clear that you can explain it to the first man whom you meet on the street." From this point of view, the representation theory is not yet complete, but it is an exciting field that has been attracting many young and enthusiastic people who will be exploring it continuously for many years, well into the 21 st century.

I would like to thank the mathematicians I met at Nankai University and Nankai Institute of Mathematics, especially Hou Zixin, Zhou Xinwei and Liang $\mathrm{Ke}$, for their hospitality during the summer school. Thanks are also due to $\mathrm{Hu}$ Sen, Lin Yang Qing, Tang Wen Jing and Wang Shu Juan of World Scientific Publishing for their professional help. Most of all, I am very grateful to David Vogan and Roger Zierau for reading the first draft of the manuscript and offering many great ideas for improvements.

Jing-Song Huang

HKUST

Clear Water Bay Hong Kong 


\section{Contents}

Preface $\quad v$

Part I. Finite Groups

Chapter 1. Finite Groups 1

$\S 1.1$. The notion of a group 1

$\S 1.2$. Groups acting on sets 3

§1.3. Finite groups 4

$\begin{array}{lll}\text { Chapter 2. Representations of Finite Groups } & 7\end{array}$

§2.1. Group representations $\quad 7$

§2.2. Characters of representations $\quad 9$

§2.3. Irreducible representations 11

$\begin{array}{lll}\text { Chapter 3. Symmetric Groups } & 15\end{array}$

§3.1. The symmetric group $S_{n} \quad 15$

§3.2. Induced representations 17

§3.3. Irreducible representations of $S_{n} \quad 19$

§3.4. Frobenius's formula $\quad 22$

§3.5. Table of characters $\quad 23$

Part II. Simple Lie Algebras

Chapter 4. Structure of Simple Lie Algebras $\quad 27$

§4.1. Basic concepts of Lie algebras $\quad 27$

§4.2. Some examples of Lie algebras $\quad 29$

§4.3. The root space decomposition $\quad 31$

§4.4. The Killing form 33

$\S 4.5$. The Weyl group $\quad 36$

§4.6. Dynkin diagrams $\quad 39$

$\S 4.7$. The classification of simple Lie algebras 44

Chapter 5. Representations of Simple Lie Algebras 47

$\begin{array}{ll}\S 5.1 . \text { Representations and modules } & 47\end{array}$ 
$\S 5.2$. Representations of $\operatorname{sl}(2, \mathbb{C})$

§5.3. Universal enveloping algebras $\quad 51$

$\S 5.4$. Verma modules

§5.5. Finite-dimensional irreducible $\mathfrak{g}$-modules $\quad 54$

$\S 5.6$. Weyl's character and dimension formulae 56

Chapter 6. Fundamental Representations $\quad 59$

$\S 6.1$. Fundamental representations $\quad 59$

§6.2. The Clifford algebra $\quad 63$

$\S 6.3$. Embedding of $s o(n, \mathbb{C})$ in the Clifford algebra 66

$\S 6.4$. Spin representations of $s o(n, \mathbb{C}) \quad 68$

\section{Part III. Compact Lie Groups}

Chapter 7. Introduction to Lie Groups $\quad 71$

§7.1. Manifolds and tangent spaces $\quad 71$

$\begin{array}{ll}\S 7.2 . & \text { Lie groups and Lie algebras } \\ & 75\end{array}$

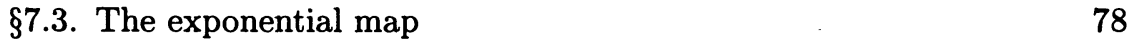

§7.4. Homogeneous spaces $\quad 83$

§7.5. Compact Lie groups and maximal tori $\quad 85$

Chapter 8. Representations of Compact Lie Groups $\quad 89$

$\S 8.1$. Representations of compact groups $\quad 89$

§8.2. Weyl's unitary trick $\quad 91$

§8.3. Representations of $S U(2)$ and $S O(3)$

§8.4. Characters $\quad 96$

$\S 8.5$. The Peter-Weyl theorem $\quad 99$

§8.6. Spherical harmonics 103

§8.7. The Borel-Weil theorem 105

$\begin{array}{lll}\text { Chapter 9. Weyl's Construction } & 109\end{array}$

§9.1. Weyl's construction for classical groups 109

§9.2. Extension of Weyl's construction to exceptional groups 113

$\begin{array}{ll}\S 9.3 \text {. Faithful irreducible representations } & 124\end{array}$

\section{Part IV. Noncompact Lie Groups}

Chapter 10. Structure of Noncompact Lie Groups 129

$\S 10.1$. Linear reductive groups and Cartan decomposition $\quad 129$

$\S 10.2$. Other decompositions $\quad 130$

§10.3. Real simple Lie algebras and Riemannian symmetric spaces 133

Chapter 11. Representations of Noncompact Lie Groups 139

$\S 11.1$. Representations and $(\mathfrak{g}, K)$-modules 
$\S 11.2$. Irreducible $(\mathfrak{g}, K)$-modules of $S L(2, \mathbb{R}) \quad 141$

§11.3. Global characters $\quad 147$

$\S 11.4 . \tau$-spherical eigenfunctions $\quad 150$

$\S 11.5$. The dimension of the space of $\tau$-spherical eigenfunctions $\quad 153$

$\S 11.6$. Global bases of the space of $\tau$-spherical eigenfunctions $\quad 156$

$\S 11.7$. Character formulas of spaces of eigenfunctions of $Z(\mathfrak{g}) \quad 159$

Chapter 12. Nilpotent Orbits and Minimal Representations 163

$\S 12.1$. Introduction to minimal representations $\quad 163$

$\begin{array}{ll}\S 12.2 \text {. Nilpotent coadjoint orbits } & 165\end{array}$

§12.3. Joseph ideals and minimal representations $\quad 169$

§12.4. Unitarity of minimal representations $\quad 171$

$\S 12.5$. Shared orbit pairs and branching rules $\quad 173$

$\begin{array}{ll}\S 12.6 . \text { Dual pair correspondences } & 175\end{array}$

$\begin{array}{ll}\S 12.7 . \text { Proof of the key lemma } & 179\end{array}$

$\begin{array}{ll}\text { Bibliography } & 185\end{array}$ 